\title{
Erratum: Folding of Toll-like receptors by the HSP90 paralogue gp96 requires a substrate-specific cochaperone
}

Bei Liu, Yi Yang, Zhijuan Qiu, Matthew Staron, Feng Hong, Yi Li, Shuang Wu, Yunfeng Li, Bing Hao, Robert Bona, David Han \& Zihai Li

Nature Communications 1:79 doi: 10.1038/ncomms1070 (2010); Published 21 Sep 2010; Updated 31 Jan 2012.

The image in Figure 5d of this Article was inadvertently duplicated from panel e. The correct version of the figure appears below.

a

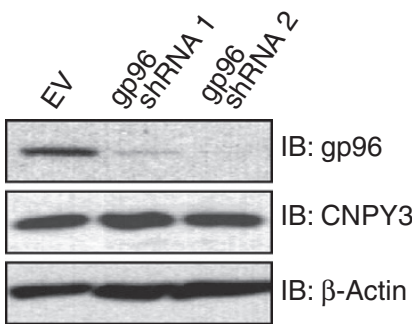

b $\quad-\quad+-$ Endo $\mathrm{H}$
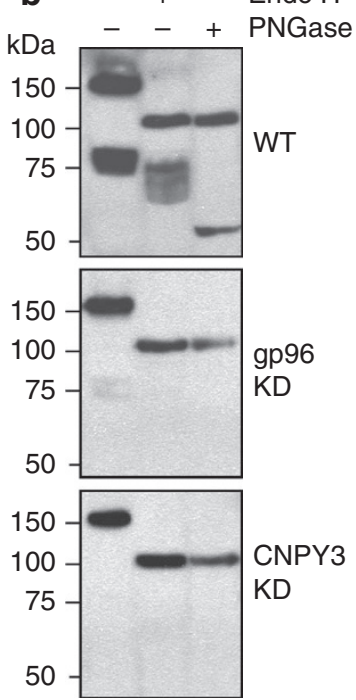

C

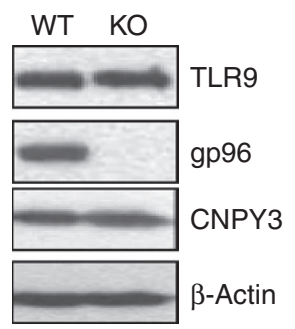

$$
\text { IP: ISO Flag } \frac{\text { WT }}{\text { ISO Flag }}
$$
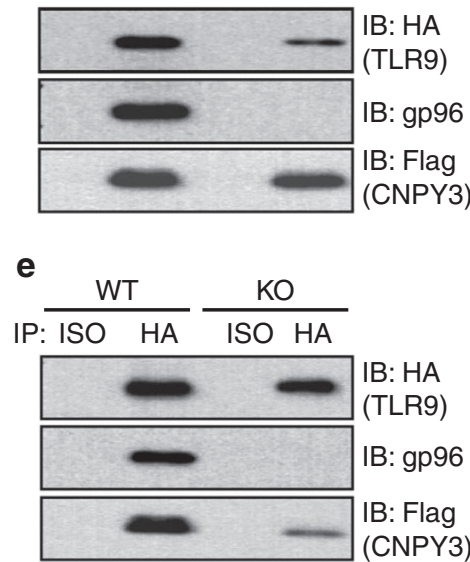